\title{
Novel insight into Chronic Inflammatory Demyelinating Polineuropathy in APECED syndrome: molecular mechanisms and clinical implications in children
}

\author{
Mariella Valenzise ${ }^{1 *}$, Tommaso Aversa$^{1}$, Giuseppina Salzano ${ }^{1}$, Giuseppina Zirilli', Filippo De Luca ${ }^{1}$ and Maureen Su²
}

\begin{abstract}
Autoimmune polyendocrinopathy-candidiasis-ectodermal-dystrophy (APECED) is a rare primary immunodeficiency disorder typically caused by homozygous AIRE gene mutation. It is characterized by the association of multiple autoimmune diseases, with a classical triad including chronic mucocutaneous candidiasis, hypoparathyroidism and adrenocortical failure. Its clinical spectrum has significantly enlarged in the last years with the apparence of new entities. One of these novel manifestations is the chronic inflammatory demyelinating polineuropathy (CIDP), that is characterized by involvement of peripheral nervous system, with nerve demyelination, progressive muscular weakness of both arms and legs and sensory loss. The identification of myelin protein zero as an important autoantigen (Ag) in CIDP may suggest the development of Ag-based therapies, such as Ag-specific DNA vaccination or infusion of Ag-coupled cells.
\end{abstract}

Keywords: AIRE gene, Autoimmune polyendocrinopathy-candidiasis-ectodermal-dystrophy, Molecular pathophysiology, Peripheral neuropathy, Specific therapies

\section{Background}

Autoimmune polyendocrinopathy-candidiasis-ectodermaldystrophy (APECED) is a rare primary immunodeficiency disorder, which is typically caused by homozygous AIRE gene mutations $[1,2]$. It is a T-cell mediated disease with increased frequencies of $\mathrm{CD} 8+$ effectors and reduction of FoxP3 $+\mathrm{T}$ regulatory cells. In addition, APECED patients show a significant alteration of the B-cell phenotype and a dysregulation of the B-cell function involving peripheral innate immune mechanisms, particularly those with longer disease duration [3].

APECED is characterized by the association of multiple autoimmune diseases, with a classical triad including chronic mucocutaneous candidiasis, hypoparathyroidism and adrenocortical failure. Its clinical spectrum has significantly enlarged in the last years [4]. In fact, besides the classical triad, many other endocrine and non-endocrine

\footnotetext{
* Correspondence: mvalenzise@unime.it

'Department of Human Pathology of Adulthood and Childhood, University of Messina, Via Consolare Valeria-98125, Messina, Italy

Full list of author information is available at the end of the article
}

autoimmune manifestations, several of which are associated with significant morbidity or mortality, may occur in this condition and vary significantly, even inside the same families and in children with the same AIRE mutations [5-7]. One of these novel diseases, i.e., the chronic inflammatory demyelinating polineuropathy (CIDP), is characterized by an involvement of peripheral nervous system with nerve demyelination, progressive muscular weakness of both arms and legs and sensory loss [7].

The aims of this review are: 1) to highlight the molecular aspects of CIDP in mice and humans; 2) to report the most recent views on its pathogenesis and 3) to alert pediatricians to the unusual neurological signs of the disease that may suggest CIDP diagnosis in children.

\section{Mechanisms of Aire action}

Aire promotes $\mathrm{T}$ cell tolerance to self-antigens by upregulating the ectopic expression of a wide array of tissuespecific self-antigens in medullary thymic epithelial cells (mTECs) within the thymus [8]. Upregulation of selfantigens in mTECs promotes the deletion of developing 
$\mathrm{T}$ cells that recognize these self-antigens with high affinity (Fig. 1a). In patients and mice with mutations in AIRE, loss of Aire function results in decreased expression of tissue-specific self-antigens by mTECs and defective negative selection of self-reactive $\mathrm{T}$ cells (Fig. 1b) [9]. Escape of these self-reactive $T$ cells into the periphery predisposes to the development of autoimmunity against multiple organs.

In addition to its role in the negative selection of effector $\mathrm{T}$ cells, Aire has been implicated also in other functions, which may promote self-tolerance. For example, Aire has been postilated to play a role in antigen presentation within the thymus [9] and to affect the frequency and function of regulatory $\mathrm{T}$ cells (Tregs), which are $\mathrm{T}$ cell subsets with immunosuppressive function. Thus, Aire may prevent autoimmunity by affecting a number of immunoregulatory mechanisms.

Since the translated Aire protein is involved in the transcriptional regulation of the expression of organspecific antigens within the thymus, the fundamental pathogenetic mechanism, in APECED patients, appears to be $\mathrm{T}$ cell mediated [10].

\section{NOD mice heterozygous for the Aire G228W mutation develop spontaneous autoimmune peripheral polyneuropathy}

A link between Aire deficiency and the development of autoimmune peripheral neuropathy was made in mice harboring the AIRE G228W mutation on the autoimmuneprone non-obese diabetic (NOD) background. The AIRE G228W point mutation was originally described in an Italian kindred with Mendelian inheritance of autoimmunity and differs from most AIRE mutations in its dominant inheritance pattern and distinct pattern of autoimmunity. To study the effects of this mutation on Aire function, we engineered knockin mice with the G228W mutation targeted by homologous recombination to the AIRE locus [11]. Similar to patients with one copy of this mutation, heterozygous mice (Aire ${ }^{G W /+}$ mice) develop spontaneous autoimmunity in a pattern distinct from Aire ${ }^{o / o}$ mice. Aire $e^{G W /+}$ mice have partial loss of Aire function in that mTECs retain approximately $10 \%$ of normal levels of tissue-specific self-antigen expression . This residual antigen expression appears to be sufficient to protect from certain autoimmune diseases that develop in Aire ${ }^{o / o}$ mice, including those associated with early lethality on the NOD background. Survival after the first months may allow development of autoimmune manifestations with later onset, including spontaneous autoimmune peripheral polyneuropathy (SAPP).

Of note, the dominant AIRE G305S mutation has also been associated with the development of neuropathy in a patient cohort [12]. It is important to underline, however, that patients with two copies of AIRE mutations have also been noted to develop neuropathy. Thus, the development of autoimmune peripheral neuropathy does not appear to be specific to dominant AIRE mutations but can occur in patients who have either one dominant AIRE mutation or two copies of AIRE mutations.

\section{SAPP in NOD.Aire ${ }^{G W /+}$ mice shares multiple features with CIDP in humans}

By 20 weeks of age, approximately $80 \%$ of female Aire $e^{G W /+}$ mice on the NOD background (NOD.Aire ${ }^{G W /+}$ mice)

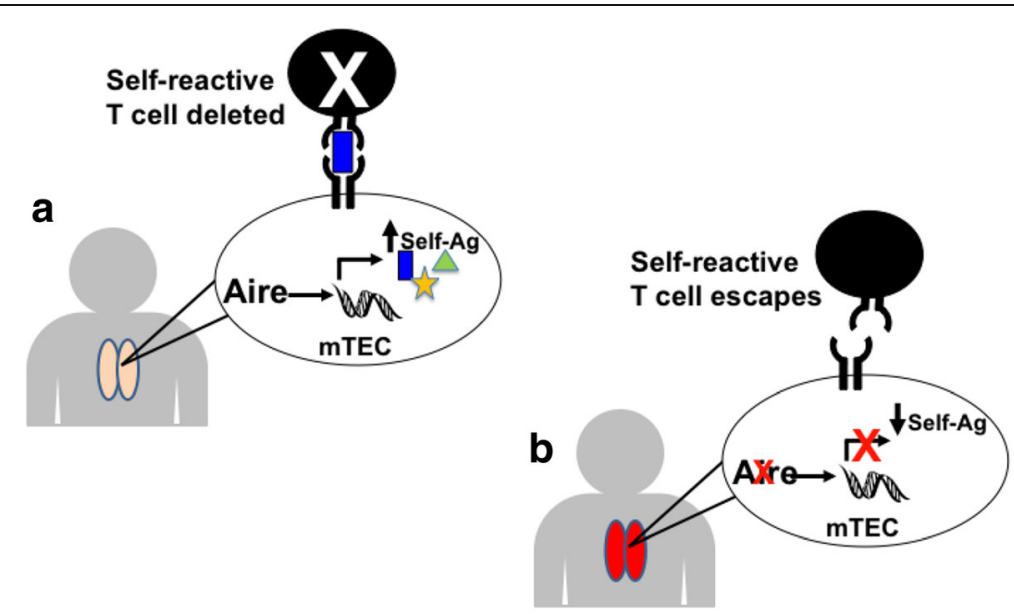

Fig. $1 \mathbf{a}$ and $\mathbf{b}$ Aire promotes expression of self-antigens in mTECs and deletion of self-reactive T cells within the thymus. a In a normal thymus, Autoimmune Regulator (Aire) upregulates expression of a large number of tissue-specific self-antigens (Self-Ag) within medullary thymic epithelial cells (mTECs). Developing $T$ cells which recognize these self-antigens with high affinity are eliminated to prevent these $T$ cells from causing autoimmunity. $\mathbf{b}$ In Aire deficient individuals, lack of Aire expression within mTECs results in decreased expression of tissue-specific self-antigens in mTECs. Lack of presentation of these self-antigens to self-reactive T cells allows escape of these self-reactive $T$ cells from clonal deletion. Release of self-reactive $T$ cells into the periphery promotes autoimmunity 
develop spontaneous neuropathy $[11,13]$ that is not seen in wildtype (NOD.WT) littermates. Affected mice display bilateral weakness of the hind limbs that may subsequently progresses to severe paralysis of all limbs. This neuropathy is associated with immune cell infiltration in NOD.Aire ${ }^{G W /+}$ sciatic nerves, but not in brain or spinal cord.

In order to demonstrate the importance of immune cells in the pathogenesis of this neuropathy, we transferred spleen and lymph node cells from neuropathic NOD.Aire ${ }^{G W /+}$ mice into immunodeficient NOD Prkds $c^{\text {scid/scid }}$ (NOD.scid) mice. All recipients developed

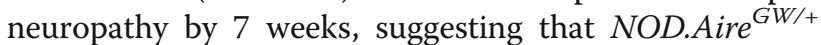
immune cells are pathogenic [13]. Furthermore, recipients of purified CD4+ T cells also developed neuropathy that was accompanied by immune cell infiltration of sciatic nerves [12], suggesting that CD4+ $\mathrm{T}$ cells are sufficient to transfer SAPP.

SAPP in NOD.Aire ${ }^{G W /+}$ mice shares a number of features with CIDP. First, CD4+ T cells and F4/80+ macrophages are abundant immune cell types in CIDP patient nerve biopsies. Similarly, CD4+ T cells and F4/80+ macrophages are also prevalent in the immune infiltrates of NOD.Aire ${ }^{G W /+}$ sciatic nerves [13]. Second, CD4+ helper T cells in CIDP patients are skewed toward the production of interferon (IFN) gamma. Similarly, approximately $40 \%$ of CD4+ helper $\mathrm{T}$ cells in the sciatic nerve infiltrates of neuropathic NOD.Aire ${ }^{G W /+}$ produce IFN gamma after in vitro stimulation and less than $1 \%$ produce IL-17 or IL-4 [12]. Third, increased expression of the chemokine CXCL10 (IP-10) in sural nerve biopsies is associated with CIDP. In neuropathic NOD.Aire ${ }^{G W /+}$ mice, CXCL10 expression is also increased in the sciatic nerves. Finally, demyelination is a hallmark of CIDP, and extensive demyelination is characteristic of sciatic nerves from neuropathic NOD.Aire $e^{G W /+}$ mice [13].

Thus, SAPP in this mouse model recapitulates a number of features of human CIDP.

\section{Defective central tolerance to PO underlies the immune tolerance defect in NOD.Aire ${ }^{G W /+}$ mice}

A key question in autoimmune disease is the identity of inciting antigens. This question has been particularly difficult to address in CIDP because only a minority of patient sera samples contain PNS antigen-specific autoantibodies. Aire deficiency, on the other hand, has been associated with autoantibody production against multiple organs in both mice and humans [7]. We therefore utilized NOD.Aire ${ }^{G W /+}$ mice as a strategy to identify potential relevant peripheral nerve antigens important in SAPP [13]. Using autoantibodies present in the sera of neuropathic NOD.Aire ${ }^{G W /+}$ mice, we isolated an antigen that appeared to be recognized by all PNS-reactive serum specimens from NOD.Aire ${ }^{G W /+}$ mice. This antigen was identified by mass spectrometry to be Myelin Protein zero (P0), a $28 \mathrm{kD}$ protein which compromises the majority of peripheral nerve myelin.

$\mathrm{PO}$ is a known autoantigen in PNS autoimmunity in various other settings. Although autoantibodies against PNS antigens occurs in only a minority of CIDP patients, the most frequent autoantibody specificity is against P0. In mice other animals, immunization with P0 is sufficient to provoke Experimental Autoimmune Neuropathy (EAN), an induced model of autoimmune peripheral neuropathy that has been widely used to study mechanisms of disease. Finally, P0 has been identified as an important target antigen in spontaneous PNS autoimmunity which develops in NOD mice deficient for the costimulatory molecule B7-2, and NOD mice engineered to express T cells with specificity against P0 develop early onset, fulminant neuropathy.

Based on our working model of Aire action (Fig. 1), we hypothesized that $\mathrm{PO}$ is also expressed within mTECs and therefore promotes clonal deletion of $\mathrm{P} 0$ specific $\mathrm{T}$ cells developing within the thymus. Furthermore, we hypothesized that thymus expression of P0 would be under the control of Aire, so that decreased P0 expression would be detected in mTECs from Aire deficient mice. In support of this, P0 expression was indeed detectable within mTECs and detected at approximately $10 \%$ of wildtype levels in mTECs derived from NOD.Aire $e^{G W /+}$ mice [12]. Additionally, as would be expected if lack of clonal deletion was occurring in Aire-deficient thymus, increased $\mathrm{T}$ cell reactivity to $\mathrm{PO}$ was seen in the spleen

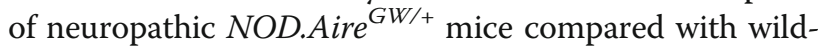
type counterparts [12]. Thus, lack of Aire-mediated expression of P0 in mTECs is associated with increased immune response to $\mathrm{P} 0$ and development of spontaneous autoimmunity against the PNS.

\section{PO is also an autoantigen in APECED patients with CIDP}

In people, autoimmune peripheral neuropathy manifests most commonly as CIDP or Guillan Barre Syndrome. These diseases occur with a combined prevalence of between 2 and 8 per 100,000. The pathophysiology of autoimmune peripheral neuropathy was not well understood, and, importantly, the pathogenic specific antigen of autoimmune peripheral neuropathy was not known. In fact, if there is general agreement that CIDP is an autoimmune disorder, significant questions remain concerning disease mechanisms and target antigens.

CIDP typically presents as a progressive or relapsing, symmetrical mainly motor neuropathy with proximal and distal weakness. The diagnosis of CIDP usually depends upon the electrophysiological studies showing features consistent with demielination as prolongation of distal latency, or reduction in motor conduction velocity, or prolongation of $\mathrm{F}$ wave latency, or absence of $\mathrm{F}$ waves, or partial conduction block, or abnormal temporal 
dispersion, or distal CMAP duration and one other demyelinating parameter in one other nerve. Supportive features for a diagnosis of CIDP include: elevated cerebrospinal fluid proteins without an increased leukocyte count; nerve biopsy finding of primary demyelination or improvement following immunotherapy.

As mentioned previously, two unrelated patients with mutations in AIRE also spontaneously developed CIDP.

The boys described by our group [7] showed progressive muscular weakness involving both arms and legs, which was associated with sensory loss and absent tendon reflexes.

Electromyograhy (EMG) revealed neurogenic changes with denervation in the intrinsic muscles of the hands. Motor and sensory nerve conduction velocities were reduced in both ulnar nerves and in the right median nerve, with signs of chronodispersion.

Sural nerve biopsy, performed in one of these patients showed moderate demyelination in all nerve fascicles, patchy CD4-, CD8- and CD68-positive cell infiltration around two small endoneural blood vessel walls and moderate subperineural oedema.

No serum autoantibodies against either myelinassociated glycoprotein or peripheral nerve myelin proteins (GM1 gangliosides and onco-neural antigens-HU), CV2, amphyphysin) [6]. By radioligand binding assay, however, both of these patients demonstrated autoantibodies against P0, suggesting that P0 is an important antigen in both mice and humans deficient in Aire [13].

Current treatments consist primarily of glucocorticoids, intravenous immunoglobulin (I.V. Ig) and plasmapheresis, which result in non-specific immunosuppression. Recently, subcutaneous immunoglobulins (SC-Ig) has received increasing attention because its efficacy seem to be similar to I.V.Ig [14-16]. In those patients, neurological symptoms and electrophysiological tests significantly improved after an initial therapy with I.V.Ig ( $400 \mathrm{mg} / \mathrm{kg}$ per day for 5 days; therapy regimen was repeated every 3 weeks for two months) followed by one with oral prednisone. After relapse the patient two has started treatment with SCIg with an increase in autonomy and quality of life through self treatment. Despite this advance with SCIg, more specific therapies that target PNS autoimmunity remains an unmet clinical need.

\section{Conclusions}

1) MP0 is a target of autoimmunity in APECED children;

2) therefore, AIRE-mediated autoimmune peripheral neuropathy should be included among the components of this syndrome;

3) the identification of P0 as an important autoantigen in CIDP may suggest the development of Ag-based therapies, such as Ag-specific DNA vaccination or infusion of Ag-coupled cells;

4) the demonstration of the altered B cell phenotype in APECED patients may suggest the possible utility of future anti B-cell treatments such as Rituximab in order to control an underlying immunological defect therefore avoid the disease progression to multiorgan autoimmunity.

\section{Abbreviations}

AIRE: Autolmmune REgulator; APECED: Autoimmune polyendocrinopathycandidiasis-ectodermal-dystrophy; CIDP: Chronic inflammatory demyelinating polineuropathy; CMAP: Compound muscle action potentials; EAN: Experimental autoimmune neuropathy; EMG: Electromyograhy; GBS: Guillan Barre Syndrome; IVIG: Intravenous immunoglobulins; MPO: Myelin protein zero; mTECs: Medullary thymic epithelial cells; NOD: Non-obese diabetic; PNS: Peripheral nervous system; SAPP: Spontaneous Autoimmune Peripheral Polyneuropathy; SClg: Subcutaneous immunoglobulin; Tregs: Regulatory T cells

Acknowledgements

No Acknowledgments are provided.

Funding

No funding was necessary considering that the present one is a Commentary.

\section{Availability of data and materials}

There is availability of our data and material.

\section{Authors' contributions}

MV and MS have written the paper; FDL and TA have organized the material and prepared its distribution in the different sections; GS and GZ have collected references. Each Author listed on the manuscript has seen and approved the submission of the present version of the manuscript and takes full responsibility for the manuscript

\section{Competing interests}

The authors declare that they have no competing interest.

\section{Consent for publication}

The informed consent has been obtained from the parents of our patients.

Ethics approval and consent to participate

The studies cited in the Commentary were approved by our Ethics Committee and all the informed consents have been obtained from the parents of patients.

\section{Author details}

${ }^{1}$ Department of Human Pathology of Adulthood and Childhood, University of Messina, Via Consolare Valeria-98125, Messina, Italy. ${ }^{2}$ Department of Microbiology and Immunology- UNC Hospitals Children's Specialty Clinic- Chapel Hill, Chapel Hill, NC, USA

Received: 8 November 2016 Accepted: 14 January 2017 Published online: 19 January 2017

\section{References}

1. Cervato S, Mariniello B, Lazzarotto F, Morlin L, Zanchetta R, Radetti G, et al. Evaluation of the autoimmune regulator (AIRE) gene mutations in a cohort of Italian patients with autoimmune-polyendocrinopathy-candidiasis-ectodermaldystrophy (APECED) and in their relatives. Clin Endocrinol. 2009;70(3):421-8.

2. Valenzise M, Wasniewska M, Mirabelli S, De Luca F, Cervato S, Betterle C. Identification of two novel mutations in the first Sicilian APECED patient with no R203X mutation in AIRE gene and review of Italian APECED genotypes. Gene. 2012;499(2):343-6.

3. Perri V, Gianchecchi E, Scarpa R, Valenzise M, Rosado MM, Giorda E et a. Altered $\mathrm{B}$ cell homeostasis and Toll-like receptor 9-driven response in patients affected by autoimmune polyglandular syndrome Type 1: Altered B cell phenotype and dysregulation of the B cell function in APECED patients. Immunobiology. 2017; 222(2):372-383. 
4. Valenzise M, Alessi L, Bruno E, Cama V, Costanzo D, Genovese C, et al. APECED syndrome in childhood: clinical spectrum is enlarging. Minerva Pediatr. 2016;68(3):226-9. 25502918.

5. De Luca F, Valenzise M, Alaggio R, Arrigo T, Crisafulli G, Salzano G, et al. Sicilian family with autoimmune polyendocrinopathy-candidiasis-ectodermal dystrophy (APECED) and lethal lung disease in one of the affected brothers. Eur J Pediatr. 2008;167(11):1283-8.

6. Valenzise M, Fierabracci A, Cappa M, Porcelli P, Barcellona R, De Luca F, et al. Autoimmune polyendocrinopathy-candidiasis-ectodermal dystrophy: report of seven additional sicilian patients and overview of the overall series from Sicily. Horm Res Paediatr. 2014;82(2):127-32.

7. Valenzise M, Meloni A, Betterle C, Giometto B, Autunno M, Mazzeo A, et al. Chronic inflammatory demyelinating polyneuropathy as a possible novel component of autoimmune poly-endocrine-candidiasis-ectodermal dystrophy. Eur J Pediatr. 2009:168(2):237-40.

8. Anderson MS, Venanzi ES, Klein L, Chen Z, Berzins SP, Turley SJ, et al. Projection of an immunological self shadow within the thymus by the aire protein. Science. 2002;298(5597):1395-401.

9. Anderson MS, Bluestone JA. The NOD mouse: a model of immune dysregulation. Ann Rev Immunol. 2005;23:447-85.

10. Fierabracci A. Recent insight into the role and molecular mechanism of the autoimmune regulator (AIRE) gene in auotimmunity. Autoimmun Rev. 2011;10:137-43.

11. Su MA, Giang K, Zumer K, Jiang H, Oven I, Rinn JL, et al. Mechanisms of an autoimmunity syndrome in mice caused by a dominant mutation in Aire. J Clin Invest. 2008;118(5):1712-26.

12. Oftedal BE, Hellesen A, Erichsen MM, Bratland E, Vardi A, Perheentupa J, et al. Dominant mutations in the autoimmune regulator AIRE are associated with common organ-specific autoimmune diseases. Immunity. 2015;42(6):1185-96.

13. Su MA, Davini D, Cheng P, Giang K, Fan U, DeVoss JJ, et al. Defective autoimmune regulator-dependent central tolerance to myelin protein zero is linked to autoimmune peripheral neuropathy. J Immunol. 2012;188(10):4906-12.

14. Danieli MG, Gelardi C, Pedini V, Moretti R, Gabrielli A, Logullo F. Subcutaneous IgG in immune-mediate diseases: proposed mechanisms of action and literature review. Autoimmun Rev. 2014:13:1182-8.

15. Markvardsen LH, Harbo T, Sindrup SH, Christiansen I, Andersen H, Jakobsen J, Danish CIDP and MMN Study Group. Subcutaneous immunoglobulin preserves muscle strength in chronic inflammatory demyelinating polyneuropathy. Eur J Neurol. 2014;21(12):1465-70.

16. Nogués MA, Varela FJ, Seminario G, Insúa MC, Bezrodnik L. Subcutaneous immunoglobulin. Treatment in chronic inflammatory demyelinating polyradiculo-neuropathy. Medicina (B Aires). 2016;76(1):36-9.

\section{Submit your next manuscript to BioMed Central and we will help you at every step:}

- We accept pre-submission inquiries

- Our selector tool helps you to find the most relevant journal

- We provide round the clock customer support

- Convenient online submission

- Thorough peer review

- Inclusion in PubMed and all major indexing services

- Maximum visibility for your research

Submit your manuscript at www.biomedcentral.com/submit

) Biomed Central 